\title{
Chiral Functionalization of Optically-Inactive Monolayer-Protected Silver Nanoclusters by Chiral Ligand-Exchange Reactions
}

\author{
Naoki Nishida, Hiroshi Yao, ${ }^{*}$ and Keisaku Kimura
}

Graduate School of Material Science, University of Hyogo, 3-2-1 Koto, Kamigori-cho, Ako-gun, Hyogo

\section{Normalized Absorption Spectra of the Fractioned Nanocluster Compounds $1_{\mathrm{D}} / \mathbf{1}_{\mathrm{L}} / \mathbf{1}_{\mathrm{rac}} / \mathbf{1}_{\mathrm{D} 50} / \mathbf{1}_{\mathrm{L} 50}$}

To further demonstrate the spectral differences among the nanocluster compounds of $\mathbf{1}_{\mathrm{D}} / \mathbf{1}_{\mathrm{L}} / \mathbf{1}_{\mathrm{rac}} / \mathbf{1}_{\mathrm{D} 50} / \mathbf{1}_{\mathrm{L} 50}$, their normalized absorption spectra are shown in Figure S1. The normalization was conducted at around $255 \mathrm{~nm}$. The spectral profiles of the $\mathbf{1}_{\mathrm{D}} / \mathbf{1}_{\mathrm{L}}\left(\right.$ or $\left.\mathbf{1}_{\mathrm{D} 50} / \mathbf{1}_{\mathrm{L} 50}\right)$ pair are quite similar with each other, whereas $\mathbf{1}_{\text {rac }}$ differs. One may say that the spectrum of $\mathbf{1}_{\mathrm{D} 50}$ would be slightly different from that of $\mathbf{1}_{\mathrm{L} 50}$, but their completely featureless profiles as well as their almost identical patterns of the anisotropy factor assure their essential equality. This slight difference probably comes from a poorer quality of the fraction $\mathbf{1}_{\mathrm{D} 50}$, because it appeared broader in the gel compared to $\mathbf{1}_{\mathrm{L} 50}$ and thus was collected with a smaller amount.
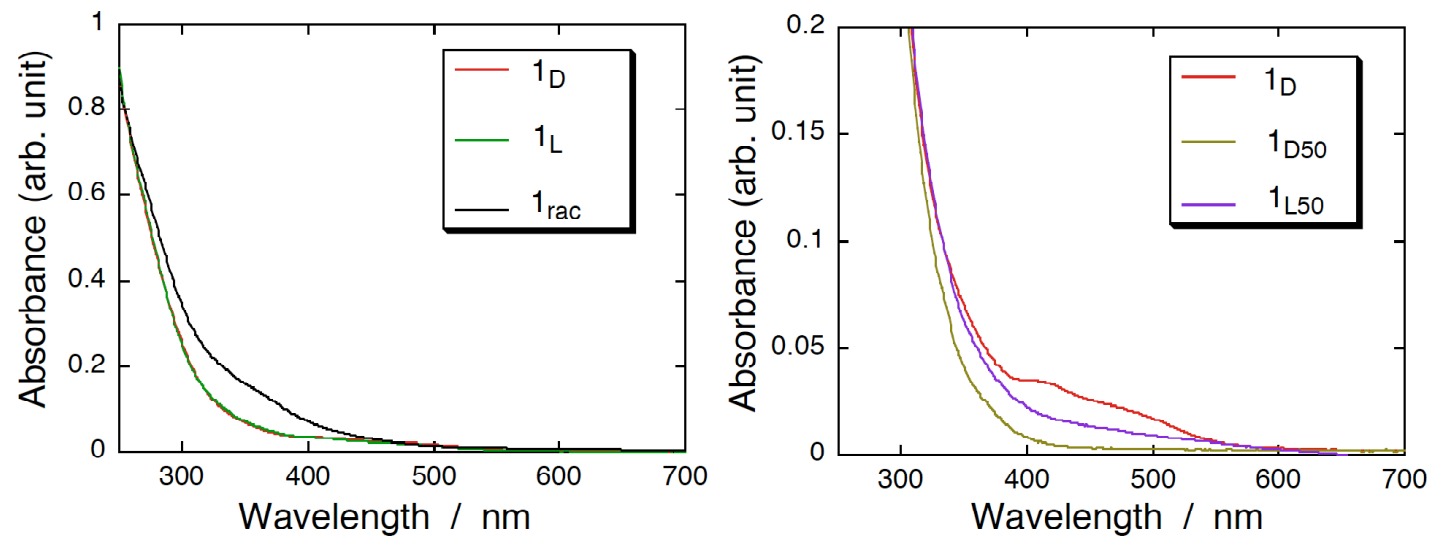

Figure S1. Normalized absorption spectra of the nanocluster compounds 1. The normalization was conducted at around $255 \mathrm{~nm}$. 
Relationship between the ee Value of the Surface Ligand and the Chiroptical Response for Compound 2

Figure S2 shows the calibration curves exhibiting a linear relationship between the enantiomeric purity of the surface ligand and the detected CD intensity normalized to the absorbance at $\sim 300 \mathrm{~nm}$ for the acid-untreated samples of $\mathbf{2}$. Although the slops were different between D-Pen- and L-Pen- protected silver nanocluster samples, an excellent linear fit could be obtained in each sample. The difference may be due to that in the stability between the compounds $\boldsymbol{2}_{\mathrm{D}}$ and $\mathbf{2}_{\mathrm{L} \cdot}{ }^{1}$ The expected positions of $\boldsymbol{2}_{\mathrm{eD}}$ and $\boldsymbol{2}_{\mathrm{eL}}$ are also shown in the figure.

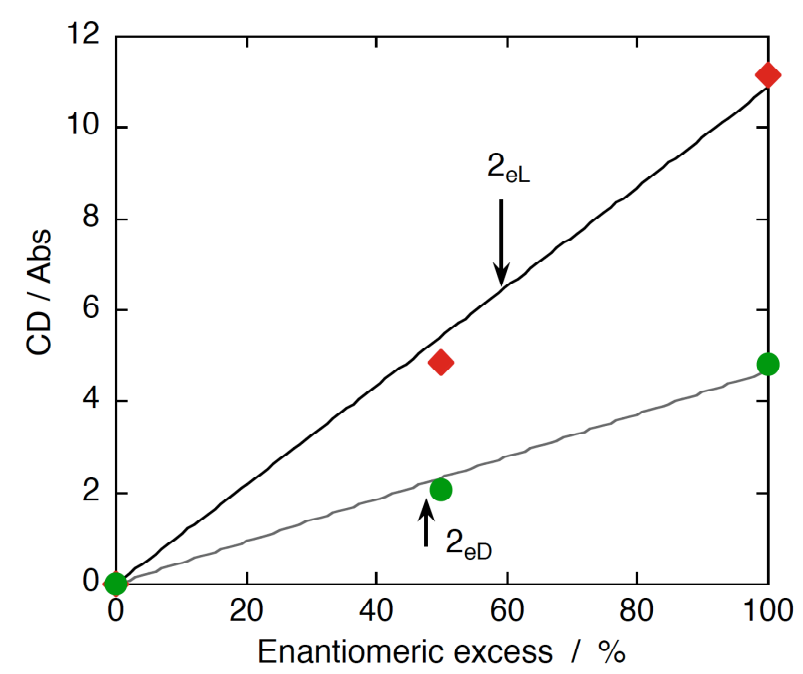

Figure S2. Relationship between the ee value of the surface ligand and the chiroptical response for compounds 2 . The plots were obtained for the compounds $\mathbf{2}_{\mathrm{D}} / \mathbf{2}_{\mathrm{D} 50}$ (circles) and $\mathbf{2}_{\mathrm{L}} / \mathbf{2}_{\mathrm{L} 50}$ (diamonds). A linear fit could be provided, respectively.

\section{References}

(1) Nishida, N.; Yao, H.; Ueda, T.; Sasaki, A.; Kimura, K. Chem. Mater. 2007, 19, 2831. 